\title{
Hypercarotenaemia as a symptom of weight phobia*
}

\author{
A. H. CRISP \\ M.D., M.R.C.P.(E), D.P.M. \\ Professor
}

\author{
E. STONEHILl \\ M.B., B.S., D.P.M. \\ Research Assistant
}

\author{
Academic Department of Psychiatry, \\ St. Georges Hospital Medical School, London, S.W.I
}

Carotenaemia is a term coined by Hess \& Myers in 1919 who described a yellow pigmentation of the skin which they attributed to the deposition of carotene. Their claim, that there is an accompanying high level of serum carotene, has since been confirmed and the condition is sometimes more accurately called hypercarotenaemia. Thus a number of laboratories, using modifications of Kimble's (1938) method of assay of carotene in the blood, regard levels between 40 and $300 \mu \mathrm{g}$ as being commonplace amongst the general population.

Hypercarotenaemia as a clinical phenomenon excited most interest several decades ago. It was described in association with a number of pathological conditions including diabetes mellitus (Niehaus, 1924 ; Rabinovitch, 1928 ; Boeck \& Yater, 1929), hypothyroidism (Stannus, 1929; Bomford, 1938; Anderson \& Soley, 1938 ; Escamilla, 1942 ; Jeghers, 1944 ; Wayne, 1960) and nephrosis (Boeck \& Yater, 1929; Josephs, 1939 ; Jeghers, 1944). It was more commonly found in association with hyperlipaemic states and Josephs (1939) suggested that this was because vitamin $\mathrm{A}$ and its precursor carotene are fat soluble. Stannus (1929) believed that hypercarotenaemia in severe diabetes mellitus and in hypothyroidism was due to a lowered metabolic rate in these conditions leading to diminished oxidation and therefore accumulation of lipochromes. Pariente et al. (1936) proposed more specifically that the conversion of carotene to vitamin A was impeded in diabetes. Anderson \& Soley (1938) suggested that carotene antagonizes thyroxine and they reported that there was some experimental evidence with rates to support this view.

Many authors, including McGill (1922), Jeghers (1944) and Abrahamson \& Abrahamson (1962), have suggested that hypercarotenaemia is harmless. However, other writers, including Sequeira (1937), Anderson \& Soley (1938) and Josephs (1939), believed that hypercarotenaemia itself pro-

\footnotetext{
*This study was conducted while the authors were working in the Academic Department of Psychiatry, Middlesex Hospital, London, W.1.
}

duced a clinical picture including weight loss, weakness and hypothyroidism. Anderson \& Soley (1938) reported on thirteen patients, seven male and six female with hypercarotenaemia. Thyroid functions were studied in seven patients, of whom five were found to have low BMRs and raised serum cholesterol levels and were judged to be hypothyroid. However, these workers also reported that some of their patients described recent weight loss and admitted to having intentionally dieted. Josephs (1939), reporting a similar clinical picture in association with hypercholesterolaemia and lowered BMR, stated that the findings were consistent with hypothyroidism but added that the loss of weight was a surprising feature.

The most important single factor contributing to the development of hypercarotenaemia is a high dietary intake of carotene-containing foods such as carrots and spinach. Many case reports have referred to an excessive consumption of such foods (Hess \& Myers, 1919; McGill, 1922; Rabinovitch, 1928; Levin \& Silvers, 1931; Josephs, 1939; Almond \& Logan, 1942; Abrahamson \& Abrahamson, 1962). There are a number of reports (Almond \& Logan, 1942 ; Abrahamson \& Abrahamson, 1962) of 'normal' subjects who developed hypercarotenaemia as a result of excessive consumption of carotene-containing foods. In particular Dagadu \& Gillman (1963) reported a high incidence of hypercarotenaemia in Ghanians and related this to dietary factors, and Ross \& Parker (1962) have commented that serum carotene levels in the general population fluctuate with the seasonal variations of availability of carotene-containing foods.

The literature on hypercarotenaemia contains many references to accompanying symptoms of a general behavioural and psychological kind. This clinical picture has often been interpreted to be the result of the hypercarotenaemia. McConaghey (1952) reported on two obese middle-aged female patients whose symptoms included depression, tiredness, lassitude and nervousness. Sequeira (1937) reported the development of hypercaroten- 
aemia in two white men living in the Tropics, at least one of whom excluded all starches from his diet. Symptoms included weakness, weight loss and hypotension. Josephs (1939) described six patients who developed hypercarotenaemia in the absence of a previously diagnosed metabolic disturbance. Two of the patients were small children: three were men and one was a woman. The latter four all had severe weight loss due to a selfimposed dietary regime involving the exclusion of carbohydrates and the ingestion of excessive amounts of carrots and spinach. Symptoms and signs included emaciation, weakness, hypotension, hypercholesterolaemia and lowered basal metabolic rate. In the female patient an idiosyncrasy to starch-containing foods was commented on. Similar symptoms were reported by Levin \& Silvers (1931) in two young unmarried women who were known to have dieted strenuously and lost much weight. One patient had been taking thyroid extract to hasten weight-loss and, having lost $28 \mathrm{lb}$, was found to have a 'nervous breakdown'. The other patient had been eating $1 \mathrm{lb}$ of carrots daily, ostensibly to hasten a cure for acne vulgaris, which had already been treated successfully.

Boeck \& Yater (1929) reviewed thirty-six patients (twenty-seven male and nine female) with xanthosis. Ten were diabetics. Xanthosis was the sole diagnosis in four. Five patients complained primarily of weakness and in three a diagnosis of chronic nervous exhaustion was made. One of these patients had been consuming $150 \mathrm{~g}$ of carrots each day.

Greene \& Blackford (1926) described fourteen cases of hypercarotenaemia and eleven of the patients were thought to be neurotic, often with somatic symptoms such as chronic indigestion and abdominal pain, but also weight loss and weakness. They alone commented that the neurosis might be a factor predisposing to the selection of the pigment-producing diet. However, they went on to say that once the dietary origin of hypercarotenaemia had been established, it was without further clinical significance.

Others have suggested that it is a simple matter to restore dietary habits towards normal in these patients and thus eliminate the hypercarotenaemia together with the other symptoms.

\section{Case report}

The following report concerns a 23-year-old woman who developed marked hypercarotenaemia. Her unusual feeding habits were based in severe chronic primary anorexia nervosa; a condition which was not immediately or readily apparent. This case is one of ninety such patients suffering with weight phobias and referred to in other contexts elsewhere (Crisp 1965a, b, 1966a, 1967). (Serial number 38 in the overall study.)

\section{Biography}

Her 53-year-old father, a bank manager, was a tall, thin, introverted, passive and dependent man. He had been away in the war during the patient's first few years of life. Subsequently he had never been able to meet the patient's childhood needs for affection; he had usually seemed to be indifferent and disinterested or else irritable and frankly rivalrous with her for the mother's affection. However, he had always showed concern that she should eat well and it was he rather than the mother who would coax her in this. The mother was an attractive, sociable, assertive character, the dominant figure in the family. Her weight and eating habits were normal. There was a 26-year-old brother who the patient had always considered to be the favoured child. He was tall and now markedly obese at $17 \frac{1}{2}$ stones. The patient insisted that their relationships had always been a friendly one and she denied that there had ever been any incest.

As a child, the patient had seemed to be a plump, boisterous, wayward tomboy. She had had a good appetite and, overall, ate well, but there had nevertheless been frequent battles, mainly with the father, over feeding. She had grown rapidly and her menarche was at the age of 11 years. At that time she was already tall, plump and big-bosomed, and was teased on these accounts. Around the same time she was sent off to the first of a series of boarding schools and finishing schools and had, thereafter, rarely lived at home. To other people at this time she continued to appear as a vigorous, frequently naughty, extrovert and unaffectionate child. However, the patient described herself as having always been nervous, shy and terrified of examinations. She eventually passed three ' $O$ ' levels in her G.C.E. At this time she showed some aptitude for Domestic Science but failed to complete the course in this subject. Subsequently she trained as a shorthand-typist.

Her menstrual bleeds had been scanty and somewhat irregular throughout her early teens. When she was 17 years of age they stopped. During her early teens she had made several unsuccessful attempts to restrict her dietary intake and lose weight. Now, at the age of 17 and 18 years she gained more weight, up to $10 \frac{1}{2}$ stones. During the next 2 years she was unable to lose weight and felt ungainly and self-conscious. Throughout her teens she had felt shy with boys, although aware she was greatly attracted to them, 
and she often wished she could flirt. At the age of 20 she attempted to diet and lose weight so as to appear more attractive and feel more confident. She lost some little weight. At this time her brother became engaged to be married. For the first time in her life she picked up a boy, an overseas student, in a cafe and during the next few weeks she had a frantic relationship with him, including frequent sexual intercourse, in which she was very frigid. She was disgusted with her behaviour but felt compelled to carry on. During these few weeks her dieting became excessive. She ate no starchy foods and existed on small quantities of salad, cheese, eggs and fruit. She lost weight from 10 to 7 stones. She became increasingly restless, concerned to take regular, frequent, strenuous exercise, suffered with increasing insomnia and constipation. She began to feel markedly cold. She lost all interest in her boyfriend. During the next year she remained in this state of clear-cut primary anorexia nervosa with sustained restriction of carbohydrate intake. However, although her brother was now married, she noticed no change in her parents' attitude towards her.

She moved into a new flat. Her neighbour was a 40-year-old man who was friendly towards her. He made no sexual demands but was concerned about her emaciated state and they spent most evenings together. During the next 3 months she gained weight up to 10 stones. When he left she became, once again, frightened and disgusted with her weight and began to vigorously restrict her carbohydrate intake again. During the next year, her eating habits pursued a regular pattern in which she would almost totally abstain from eating over several weeks and then enter a stage of 7-10 days bulimia. During these latter phases she would spend $30-40$ s a day on biscuits and cakes which she would then eat. Her weight usually fluctuated between 8 and 10 stones in association with these feeding habits. Her binges would eventually come to an end when she felt dizzy, breathless, bloated and had marked ankle oedema and swollen varicose veins. In conjunction with this she would feel increasingly disgusted with herself and would have a pervasive sense of guilt. On one such occasion while abroad she gained 4 stone in weight in 2 weeks and at the end of this time, suffered a spontaneous pneumothorax. Following this she was diagnosed as suffering with myxoedema, and was treated with thyroid medication. Her main complaint when attending doctors in the past had been of an offensive vaginal discharge. Investigation had always failed to reveal any major gynaecological pathology, and eventually some aspects of her feeding disorder became apparent.

\section{Initial examination}

Mental state. At the time of the initial interview, she had been restricting her food, and particularly her carbohydrate intake, for the past 2 weeks. She appeared as a tall, attractive, fidgety, friendly girl. She smiled readily but became tearful and agitated when describing her recurrent overwhelming impulse to eat and the attendent weight gains. She emphasized her overriding pre-occupation with diets, calories, activity and weight and when attention was directed to her nutritional history she discussed it readily enough and her account was comparable with that provided by her mother. She expressed undisguised hostility towards her father and indifference for her mother.

Physical state. Not markedly underweight. Height $5 \mathrm{ft} 8$ in; weight 8 stones $2 \mathrm{lb}$; B.P. $105 /$ 60 ; pulse rate $60 / \mathrm{min}$; cold blue extremities; loaded colon.

Investigations. Haemoblobin $90 \%$; PCV $42.5 \%$; ESR $18 \mathrm{~mm} / \mathrm{hr}$; WBC $7100 / \mathrm{mm}^{3}$, normal differential; serum cholesterol $410 \mathrm{mg} / 100 \mathrm{ml}$; serum proteins $6.5 \mathrm{~g} / 100 \mathrm{ml}$; electrophoretic strip normal pattern; serum electrolytes and magnesium normal; lactose tolerance test normal; BMR $-12 \%$ (Robertson Reid Standard); chest $X$-ray, barium meal, jejunal biopsy, faecal fat estimation and urinalysis, normal.

A diagnosis of primary anorexia nervosa was made and the patient was taken into treatment.

An attempt was made to stabilize her feeding habits as an in-patient. However, she was unable to accept foods which obviously contained carbo-

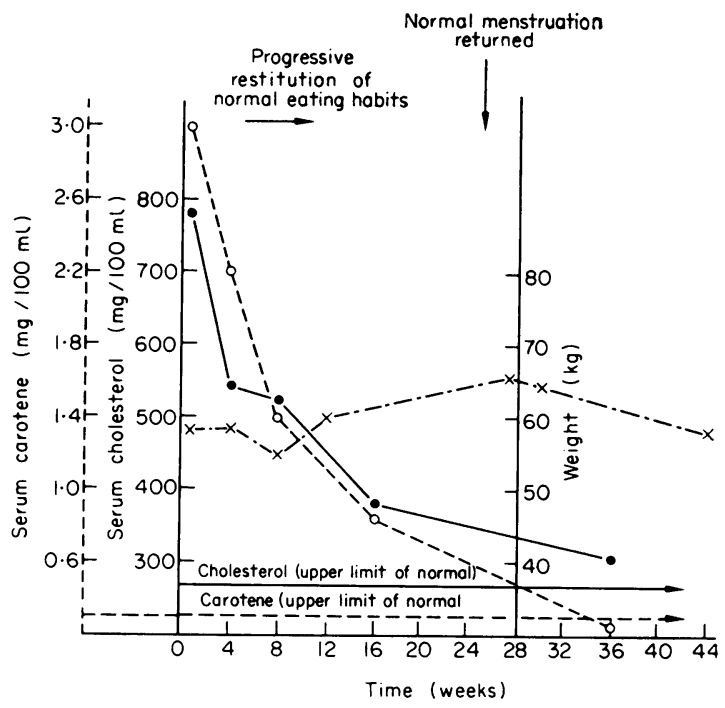

Fig. 1. Changes in serum carotene (O) and serum cholesterol $(\bullet)$ levels in relation to dietary patterns and weight changes $(x)$ in a patient with weight phobia (anorexia nervosa). 
hydrates. She was not markedly under-weight and was reluctant to accept a prolonged period of confinement to bed with strict dietary control. She started to eat regularly but sparingly and for the next year, during which time she had regular out-patient psychotherapy, she was not subject to the same bulimic episodes. Her weight remained more steady at around 8-9 stones. However, she ate mainly cheese, eggs, fruit and herring roes and continued to avoid carbohydrates. In addition she ate two large tins of spinach puree every day. After 6 months on this dietary regime, her skin appeared increasingly yellow. Her serum carotene, measured as beta carotene, was $3000 \mu \mathrm{g} / 100 \mathrm{ml}$ and her serum cholesterol was $770 \mathrm{mg} / 100 \mathrm{ml}$. At a later date, when she had accepted advice to limit her daily intake of carotene and cholesterol-containing foods, her serum beta carotene was 1300 $\mu \mathrm{g} / 100 \mathrm{ml}$, and her serum cholesterol was 520 $\mathrm{mg} / 100 \mathrm{ml}$. Recently these levels have fallen further to $250 \mu \mathrm{g}$ and $310 \mathrm{mg}$, respectively, and the yellow pigmentation of her skin has faded. She is continuing in psychotherapy aimed at modifying her weight phobia. Her menstural function has now returned and she has had four consecutive normal periods.

\section{Discussion}

Hypercarotenaemia is clearly related to excessive ingestion of carotene. As such it is an endemic feature of some societies and may vary with seasonal fluctuations in availability of carotenecontaining foodstuffs in other societies. In Western Europe and North America it has more often been reported as occurring in relation to unusual dietary habits or else has been noted to be associated with various generalized metabolic disturbances and also with hypercholesterolaemia.

The reported increased incidence amongst diabetics may, at least in part, be related to the restricted dietary habits of some such patient whereby, in association with their limited carbohydrate intake, they eat more than the usual quantity of carotene-containing foods. The diagnosis of myxoedema in association with hypercarotenaemia has often relied heavily for its justification upon findings such as a low BMR, bradycardia, hypothermia and hypercholesterolaemia. These same findings are equally indicative of severe malnutrition. The commonest cause of severe malnutrition amongst young adults in this country is probably primary anorexia nervosa, or more specifically, weight phobia. This diagnosis can often readily be made if a psychiatric history including a nutritional history is taken. However, it is a diagnosis which can otherwise easily be missed. Contrary to popular belief it is more diffi- cult to differentiate weight phobia from myxoedema than from hypopituitarism. The patient with weight phobia will not readily complain of symptoms and will usually deny or minimize major aspects of his or her illness. Indeed, such people do not usually regard themselves as ill, prefering their present state to the more threatening one of normal weight. Any complaints that they have are usually limited to such things as indigestion, constipation, swollen ankles, chilblains, insomnia or vaginal discharge. It is often important, therefore, to have an independent account.

Hypercholesterolaemia has been reported on elsewhere (Crisp, 1965a, 1966b) in association with anorexia nervosa. Crisp found that $50 \%$ of thirty female patients, with a mean age of 20 years, had serum cholesterol levels between 300 and $500 \mathrm{mg} /$ $100 \mathrm{ml}$. These high levels returned towards normal with remission of the illness. Such factors as the accompanying state of carbohydrate starvation, amenorrheoa and the associated oestrogen deficiency, the high cheese intake and the characteristic periodicity of meals displayed by these patients may contribute to this biochemical picture. However, the level of the serum cholesterol found in the present patient is exceptionally high and suggests either that this patient's dietary habits were especially evocative of both hypercarotenaemia and hypercholesterolaemia or, as has been suggested by other workers, that there is some direct interdependence between carotene and cholesterol metabolism.

\section{Conclusions}

The literature reviewed together with the evidence from the above case report suggest that when hypercarotenaemia is encountered in patients in this country it may sometimes be the result of bizarre feeding habits based in primary anorexia nervosa. Primary anorexia nervosa is regarded by the authors as a state of weight phobia leading to carbohydrate starvation. It probably has interrelated constitutional and experimental origins, and has to do with the adolescent's avoidance of her otherwise overwhelming fears of her newly found sexual impulse and the associated demands and experience of 'growing up'. It is suggested that whilst dietary advice to these patients may result in them modifying for instance their carotene intake, it is unlikely to otherwise alter the course of their illness. The latter may, however, sometimes be altered by appropriate treatment.

\section{Acknowledgments}

The authors are indebted to Dr W. Joffe, Psychoanalyst, who has conducted the psychotherapy of this patient; also to Dr L. Blendis, late Registrar, Middlesex Hospital, with whom they have had many helpful discussions. 


\section{References}

Abrahamson, I.A., SR. \& Abrahamson, I.A., JR. (1962) Hypercarotenaemia. Arch. Ophthal. 68, 4.

Almond, S. \& Logan, R.F.C. (1942) Carotenaemia. Brit. med. J. ii, 239.

Anderson, H.H. \& Soley, M.H. (1938) The effects of carotenaemia on the function of the thyroid and the liver. Amer. J. med. Sci. 195, 313.

BOECK, W.C. \& YATER, W.M. (1929) Xanthaemia and xanthosis. J. Lab. clin. Med. 14, 1129.

Bomford, R. (1938) Anaemia in myxoedema; and the role of the thyroid gland in erythropoiesis. Quart. J. Med. 7, 495.

CrISP, A.H. (1965a) Clinical and therapeutic aspects of anorexia nervosa: a study of 30 cases. J. psychosom. Res. 9, 67.

CRISP, A.H. (1965b) Some aspects of the evolution, presentation and follow-up of anorexia nervosa. Proc. roy. Soc. Med. 58, 814.

CRISP, A.H. (1966a) A treatment regime for anorexia nervosa. Brit. J. Psychiat. 112, 505.

Crisp, A.H. (1966b) Anorexia nervosa in an identical twin. Postgrad. med. J. 42, 86.

Crisp, A.H. (1967) The possible significance of some behavioural correlates weight. J. psychosom. Res. 11, 117.

Dagadu, M. \& Gillman, J. (1963) Hypercarotenaemia in Ghanaians. Lancet, i, 531.

Escamilla, R.F. (1942) Carotenaemia in myxoedema. $J$. clin. Endocr. 2, 33.
Greene, C.H. \& Blackford, L.M. (1926) Carotenaemia. Med. Clin. N. Amer. 10, 733.

Hess, A.F. \& MYers, V.C. (1919) Carotenaemia: a new clinical picture, J. Amer. med. Ass. 73, 1743.

Jeghers, H. (1944) Pigmentation of the skin. New Engl. J. Med. 228, 678.

JoSEPHS, H.W. (1939) Relation of Vitamin A and carotene to serum lipids. Bull. Johns Hopkins Hosp. 65, 112.

Kimble, M.S. (1938) The photocolorimetric determination of Vitamin A and carotene in human plasma. J. Lab. clin. Med. 24, 1055.

Levin, O.L. \& Silvers, S.H. (1931) Carotenaemia resulting from restricted diet. J. Amer. med. Ass. 96, 2190.

MCConAGHEY, R.M.S. (1952) Carotenaemia. Lancet, ii, 714.

McGill, C. (1922) Carotenaemia in an adult. J. Amer. med. Ass. 78, 281.

Niehaus, F.W. (1924) A case of carotenaemia. J. Amer. med. Ass. 82, 547.

Pariente, A.C., Present, C.H. \& Ralli, E.P. (1936) A case of carotenaemia and diabetes mellitis with necropsy report and analysis of liver for carotene, vitamin A, total fat and cholesterol. Amer. J. med. Sci. 192, 365.

RABINOVITCH, I.M. (1928) Carotenaemia and diabetes. Canad. med. Ass. J. 18, 527.

Ross, G.J. \& PARKer, J.G. (1962) Serum carotene concentration in normal individuals and its clinical interpretation. N.Y. State J. Med. 62, 3584.

Sequeira, J.H. (1937) Carotenaemia in Europeans in the tropics. Brit. J. Derm. Syph. 49, 69.

Stannus, H.S. (1929) Hyperlipochromia. Int. Clin. 1, 146. 